Check for updates

Cite this: Nanoscale Adv., 2019, 1, 140

\title{
Silver nanowire/nickel hydroxide nanosheet composite for a transparent electrode and all-solid-state supercapacitor $\dagger$
}

\author{
Haojin Du, ${ }^{a}$ Ying Pan, ${ }^{a}$ Xiao Zhang, ${ }^{a}$ Fuyang Cao, ${ }^{a}$ Tao Wan, ${ }^{a}$ Haiwei Du, (D) *b \\ Rakesh Joshi (iD a and Dewei Chu (iD *a
}

\begin{abstract}
Silver nanowire (Ag NW) based composites have shown a great potential not just in transparent electrodes but in diverse functional applications. The main challenge of Ag NW film is the large junction resistance originating from the weak NW contacts. In this paper, we report a simple method to combine ultrathin nickel hydroxide $\left(\mathrm{Ni}(\mathrm{OH})_{2}\right.$ ) nanosheets (NSs) and $\mathrm{Ag} \mathrm{NWs}$ as a composite for transparent electrode and all-solid-state supercapacitor applications. On the one hand, the $\mathrm{Ni}(\mathrm{OH})_{2} \mathrm{NSs}$ were simply coated on $\mathrm{Ag}$ NW film and the sheet resistance was decreased significantly without compromising the optical transmittance, owing to the improved junction contacts among NWs and the ultrathin nanostructure of $\mathrm{Ni}(\mathrm{OH})_{2} \mathrm{NSs}$. The optimum Ag NW/Ni(OH $)_{2}$ NS composite showed not only an excellent optoelectronic performance (a sheet resistance of $18.56 \Omega \square^{-1}$ and a transmittance of $90.26 \%$ ) but also improved thermal stability. On the other hand, the $\mathrm{Ag} \mathrm{NW/Ni(OH})_{2} \mathrm{NS}$ composite was designed for all-solid-state flexible supercapacitors with a high specific capacitance, moderate cycle stability and good mechanical flexibility, indicating a promising application in flexible supercapacitors.
\end{abstract}

Received 26th July 2018
Accepted 10th August 2018

DOI: $10.1039 / c 8 n a 00110 c$

rsc.li/nanoscale-advances

into the empty spaces among the Ag NW network which remain

\section{Introduction}

As a very promising alternative for the commercial indium tin oxides, silver nanowire (Ag NW) has been considered to be the material of next-generation transparent electrodes (TEs) in view of its large-scale industrial production and better stretching/ bending performances as well as its very good compatibility with advanced fabrication technologies such as ink-jet printing and roll-to-roll manufacturing. However, the conductivity of Ag NW networks is mainly limited by the relatively large contact resistance due to loose contacts at the nanowire junctions, and this remaining issue hinders applications somewhat. Although various post-treatments ${ }^{1}$ including thermal annealing, mechanical pressing, plasma, electron beam irradiation and laser welding are being developed to greatly reduce the wirewire junction resistance, some disadvantages such as higher temperature and costly irradiation apparatus still cannot be neglected. Compared with these welding strategies, simply depositing a coating layer on the $\mathrm{Ag} \mathrm{NW}$ film to form a hybrid or bilayer composite is very promising since the coating layer can not only strengthen the NW junction contact but also infiltrate

${ }^{a}$ School of Materials Science and Engineering, UNSW Sydney, NSW 2052, Australia. E-mail:d.chu@unsw.edu.au

${ }^{b}$ School of Chemistry and Chemical Engineering, Anhui University, Hefei 230061, P. R. China.E-mail: haiwei.du@hotmail.com

$\dagger$ Electronic supplementary information (ESI) available. See DOI: $10.1039 / \mathrm{c} 8 \mathrm{na} 00110 \mathrm{c}$ insulating and usually hinder the electron transport. Usually, the significant effects of the coating layer are (i) enlarging the contact area between Ag NWs, (ii) minimizing the surface roughness, (iii) increasing adhesion of the $\mathrm{Ag}$ NWs to the substrate, (iv) inhibiting oxidation/corrosion, and more importantly (v) facilitating electron conduction. So far, conductive materials such as PEDOT:PSS ${ }^{2}$ and graphene, ${ }^{3}$ semiconductor metal oxides ${ }^{4,5}$ and even insulating polymer matrixes like polyvinyl alcohol and chitosan, ${ }^{6,7}$ have been utilized to effectively reduce the resistance of $\mathrm{Ag} \mathrm{NW}$ electrodes. Inspired by these designs, graphene-like nanosheets (NSs) serving as a coating layer on the Ag NW network are expected to enhance the electrical conductivity of Ag NWs without sacrificing high transmittance because of the good optical performances of ultrathin NSs.

Moreover, different composites consisting of $\mathrm{Ag}$ NW and other coatings have been successfully explored to show diverse applications either in powder catalysts or in physical devices in the past decades. In particular, silver nanomaterials usually play a critical role in electrochemical energy storage and conversion devices, ${ }^{8}$ acting as a current collector or even directly participating in the electrochemical reactions. Most of the previous works have mainly focused on monofunctional applications such as electrode, biosensor or supercapacitor while multiple applications of the Ag NW based composites are highly desired (as shown in Table S1†). For example, a multifunctional $\mathrm{Ag}$ grid/PEDOT:PSS hybrid electrode was fabricated to show 
a great potential in both TE application and a solid-state supercapacitor device. ${ }^{9}$ However, the PEDOT:PSS is actually a conductive polymer and the capacitance can be further enhanced if the coating layer has a good charge-storage capacity. Unlike the conventional electric double layer capacitors (EDLCs), the charge storage in transition metal hydroxides occurs via fast and reversible redox reactions, resulting in a larger capacitance due to a dominant contribution from battery-type behavior. ${ }^{10}$ As a typical representative, nickel hydroxide $\left(\mathrm{Ni}(\mathrm{OH})_{2}\right)$ is very attractive in terms of its low cost, various morphologies, unique layered structure with a large interlayer spacing, and considerable theoretical capacitance.11

Herein, $\mathrm{Ni}(\mathrm{OH})_{2}$ NSs were coated on $\mathrm{Ag} \mathrm{NW}$ film to form a composite for a TE and all-solid-state supercapacitor. On the one hand, as a semiconductor ${ }^{12}$ like metal oxides, graphene-like ultrathin $\mathrm{Ni}(\mathrm{OH})_{2}$ NSs can effectively bridge the $\mathrm{Ag}$ NWs by filling the empty space without significantly affecting the transparency, making a contribution to optimize the optoelectronic performances. On the other hand, the excellent electrochemical performances of $\mathrm{Ni}(\mathrm{OH})_{2} \mathrm{NSs}^{\mathbf{1 3}}$ enable the composite to be a promising electrode for supercapacitor devices. The prepared composite presents a low sheet resistance $\left(R_{\mathrm{S}}=18.56\right.$ $\Omega \square^{-1}$ ) with a high optical transmittance ( 90.26\%). In addition, the all-solid-state flexible supercapacitor was fabricated by transferring the $\mathrm{Ag} \mathrm{NW} / \mathrm{Ni}(\mathrm{OH})_{2}$ NS composite onto a polydimethylsiloxane (PDMS) substrate, and the as-prepared device showed a good capacitance and mechanical flexibility.

\section{Experimental}

\section{Materials}

Silver nitrate $\left(\mathrm{AgNO}_{3}, M_{\mathrm{w}}: 169.87\right)$, polyvinylpyrrolidone (PVP) $\left(M_{\mathrm{w}}: 55000\right)$, sodium chloride ( $\mathrm{NaCl}, M_{\mathrm{w}}: 58.4$ ), 1,2-propanediol $\left(\rho: 1.036 \mathrm{~g} \mathrm{~mL}^{-1}\right)$, nickel(II) nitrate hexahydrate $\left(\mathrm{Ni}\left(\mathrm{NO}_{3}\right)_{2} \cdot 6 \mathrm{H}_{2} \mathrm{O}\right.$, $\left.M_{\mathrm{w}}: 290.81\right)$, urea $\left(M_{\mathrm{w}}: 60.04\right)$, potassium hydroxide $\left(\mathrm{KOH}, M_{\mathrm{w}}\right.$ : 56.11), polyvinyl alcohol (PVA, $M_{\mathrm{w}}: 85000-124000$ ) and carbon paper (Fuel Cell Store). All the chemicals were purchased from Sigma-Aldrich and were used without further purification.

\section{Synthesis of silver nanowires}

The synthesis of Ag NWs was conducted as reported by our previous study ${ }^{14}$ with a modification. First, $0.001 \mathrm{~g}$ NaCl was ultrasonically dissolved into $10 \mathrm{~mL}$ 1,2-propanediol. Then, $0.25 \mathrm{~g}$ PVP was put into $30 \mathrm{~mL}$ 1,2-propanediol and heated with magnetic stirring at $120{ }^{\circ} \mathrm{C}$ for $30 \mathrm{~min}$. After cooling down to $60{ }^{\circ} \mathrm{C}, 3 \mathrm{~mL} \mathrm{NaCl}$ solution was added into the solution with magnetic stirring for $3 \mathrm{~min}$. Afterwards, $12 \mathrm{~mL}$ 1,2-propanediol containing $0.3057 \mathrm{~g} \mathrm{AgNO}_{3}$ was added and the solution was heated at $150{ }^{\circ} \mathrm{C}$ with magnetic stirring for $60 \mathrm{~min}$. Finally, the as-synthesised Ag NWs were washed five times by centrifugation using ethanol and deionized (DI) water, and redispersed into ethanol.

\section{Synthesis of nickel hydroxide NSs}

First, $0.1454 \mathrm{~g} \mathrm{Ni}\left(\mathrm{NO}_{3}\right)_{2} \cdot 6 \mathrm{H}_{2} \mathrm{O}$ was dissolved into $2.5 \mathrm{~mL}$ DI water with magnetic stirring for $3 \mathrm{~min}$. Then $10 \mathrm{~mL} \mathrm{1,2-}$ propanediol and $0.12 \mathrm{~g}$ urea were added. Afterwards, the obtained solution was transferred into a Teflon container and hydrothermally heated at $150{ }^{\circ} \mathrm{C}$ for $12 \mathrm{~h}$. After cooling down to room temperature, the as-synthesised $\mathrm{Ni}(\mathrm{OH})_{2}$ NSs were washed three times by centrifugation using ethanol and DI water, and redispersed into DI water. Finally, the $\mathrm{Ni}(\mathrm{OH})_{2}$ NSs were ultrasonically exfoliated for $30 \mathrm{~min}$ (SONICS 750 Watt ultrasonic processor), and dispersed in DI water at a concentration of $0.12 \mathrm{mg} \mathrm{mL}^{-1}$.

\section{Fabrication of silver NW/nickel hydroxide TEs}

First, glass slides $\left(2 \times 2 \mathrm{~cm}^{2}\right.$, thickness of $\left.1.6 \mathrm{~mm}\right)$ were cleaned by ethanol and DI water, and treated by the UV/Ozone surface processor (Sen Lights Corporation, Japan) for $5 \mathrm{~min}$. Then, the Ag NW solution was spin coated several times onto the glass slides. Afterwards, $20 \mu \mathrm{L}$ solution containing the $\mathrm{Ni}(\mathrm{OH})_{2} \mathrm{NSs}$ was drop coated 1-5 times onto the $\mathrm{Ag} \mathrm{NW}$ films. The repeating coating cycles were conducted after the films were dried at room temperature. The schematic of TE fabrication is depicted in Scheme 1a.

\section{Synthesis of the solid electrolyte and PDMS}

First, $0.5 \mathrm{~g}$ PVA was dispersed into $5 \mathrm{~mL}$ DI water and then heated at $95{ }^{\circ} \mathrm{C}$ under magnetic stirring until the PVA was completely dissolved. Second, $1 \mathrm{~mL} 12.5 \mathrm{M} \mathrm{KOH}$ solution was added dropwise into the viscous PVA solution under magnetic stirring and the solution was dried until a gel was obtained. The gel was placed in a vacuum box to remove the gas bubbles. For the flexible device, PDMS (Sylgard 184, DowCorning) was prepared by pouring a mixture of base and curing agent (10:1 by weight) into a mould.

\section{Fabrication of silver NW/nickel hydroxide NS based all-solid- state supercapacitors}

Ag NWs were coated onto a glass substrate to achieve a low resistance (less than $5 \Omega$ ). Then the PDMS liquid was poured onto the Ag NW film, and cured at $80{ }^{\circ} \mathrm{C}$ for $1 \mathrm{~h}$. After being peeled off, the $\mathrm{Ag}$ NWs were transferred onto the PDMS

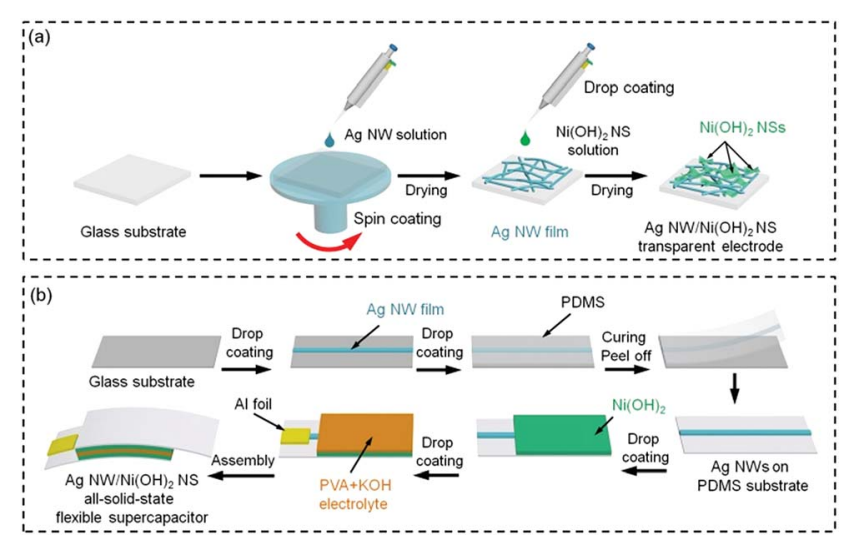

Scheme 1 Schematic of the fabrication of (a) the $\mathrm{Ag} \mathrm{NW} / \mathrm{Ni}(\mathrm{OH})_{2} \mathrm{NS}$ $\mathrm{TE}$ and (b) the all-solid-state flexible supercapacitor. 
substrate. The $\mathrm{Ni}(\mathrm{OH})_{2}$ NS solution as the supercapacitive material was drop coated onto the $\mathrm{Ag}$ NW electrode. After drying at room temperature, $\mathrm{PVA} / \mathrm{KOH}$ gel was coated onto the $\mathrm{Ni}(\mathrm{OH})_{2}$ NS layer. Finally, two pieces of electrode were oppositely assembled to fabricate a sandwich structure, and the all-solid-state supercapacitor device was dried at $60{ }^{\circ} \mathrm{C}$ to evaporate the excess water. Then two aluminium foils were pasted onto the electrodes for electrochemical tests. The schematic of the supercapacitor device fabrication is depicted in Scheme $1 \mathrm{~b}$.

\section{Materials and device characterization}

Structural analysis of the as-synthesized $\mathrm{Ag} \mathrm{NWs}$ and $\mathrm{Ni}(\mathrm{OH})_{2}$ NSs was performed using an X-ray diffractometer with $\mathrm{Cu} \mathrm{K} \alpha$ radiation $(\lambda=0.1541 \mathrm{~nm})$. The microstructures were observed by transmission electron microscopy (TEM, FEI Tecnai G2) and scanning electron microscopy (SEM, FEI Nova NanoSEM 450). The sheet thickness of $\mathrm{Ni}(\mathrm{OH})_{2}$ was measured by atomic force microscopy (AFM, Bruker) using a Scanasyst probe. The chemical bonding states were determined by X-ray photoelectron spectroscopy (XPS, ESCALAB250Xi spectrometer). The transmittance of the TEs was determined using a PerkinElmer UVvisible spectrometer. The $R_{\mathrm{S}}$ values were determined using a Four-point Probe Resistance Tester (Zhuhai Kaivo Optoelectronic Technology Co., Ltd.). The electrochemical performance was investigated using the electrochemical workstation (Autolab PGSTAT302 N).

\section{Results and discussion}

Fig. 1 shows the morphologies of $\mathrm{Ag} \mathrm{NWs}$ and $\mathrm{Ni}(\mathrm{OH})_{2}$ NSs. Similar to the results in our previous work, ${ }^{14}$ the Ag NWs have a relatively uniform length and diameter, as shown in Fig. 1a-c. The as-synthesized $\mathrm{Ni}(\mathrm{OH})_{2}$ shows a free-standing 2D NS morphology (Fig. 1d) with a lateral size ranging from submicrometers to a few micrometres, and the wrinkled surface can be clearly seen in the TEM image (Fig. 1e). The selected area electron diffraction (SAED) pattern taken from the NSs is composed of two diffraction rings corresponding to the (100) and (110) planes of $\alpha-\mathrm{Ni}(\mathrm{OH})_{2}$ (inset of Fig. 1e). Fig. 1f shows the SEM image of exfoliated $\mathrm{Ni}(\mathrm{OH})_{2}$ NSs. After the ultrasonic treatment, the large NSs have been exfoliated to many smaller nanoplatelets of a more uniform size, and the NSs are also flatter. As a result of the size reduction, the exfoliated $\mathrm{Ni}(\mathrm{OH})_{2}$ NSs form a well-dispersed colloidal suspension in water, as indicated by the clear Tyndall light scattering (inset photograph in Fig. 1f). The ultrathin and flat NS structure is also revealed by TEM (Fig. 1g). In order to determine the thickness of the NSs, the exfoliated $\mathrm{Ni}(\mathrm{OH})_{2}$ NSs were transferred onto a silicon substrate for AFM characterization (as shown in Fig. 1h) and the measured thickness from height profile is around $6 \mathrm{~nm}$, indicating a very thin NS structure. Fig. 1i shows the Raman spectrum of the $\mathrm{Ni}(\mathrm{OH})_{2}$ NSs and the broad peak located at around $500 \mathrm{~cm}^{-1}$ is indicative of a defective or disordered $\mathrm{Ni}(\mathrm{OH})_{2} \cdot{ }^{15}$

Fig. 2a presents the XRD patterns of $\mathrm{Ni}(\mathrm{OH})_{2} \mathrm{NSs}, \mathrm{Ag} \mathrm{NWs}$, and the $\mathrm{Ag} \mathrm{NW} / \mathrm{Ni}(\mathrm{OH})_{2} \mathrm{NS}$ composite. The as-synthesized $\mathrm{Ag}$ NWs display two main diffraction peaks that are well matched to the Ag standard peaks (JCPDS no. 04-0783). The diffraction peaks of the $\mathrm{Ni}(\mathrm{OH})_{2}$ NSs can be indexed to $\alpha-\mathrm{Ni}(\mathrm{OH})_{2}$ (JCPDS no. 22-0444), and no secondary phase is observed. Meanwhile, the (001) diffraction peak has a very high intensity and the intensity ratio of $I_{(001)} / I_{(110)}$ is remarkably large compared with that of the standard card. Since the $\alpha-\mathrm{Ni}(\mathrm{OH})_{2}$ has a hexagonal layered structure, the $\mathrm{Ni}(\mathrm{OH})_{2}$ NSs show anisotropic growth with a preferential direction along the $a b$ plane, ${ }^{\mathbf{1 6}}$ resulting in a 2D NS structure, which is consistent with the microstructure observation (Fig. 1e and f). The composite contains both Ag NWs and $\mathrm{Ni}(\mathrm{OH})_{2}$ NSs without any structural change. The composition and surface state of the composite were further characterized by XPS (Fig. 2b-d), in which the impurities such
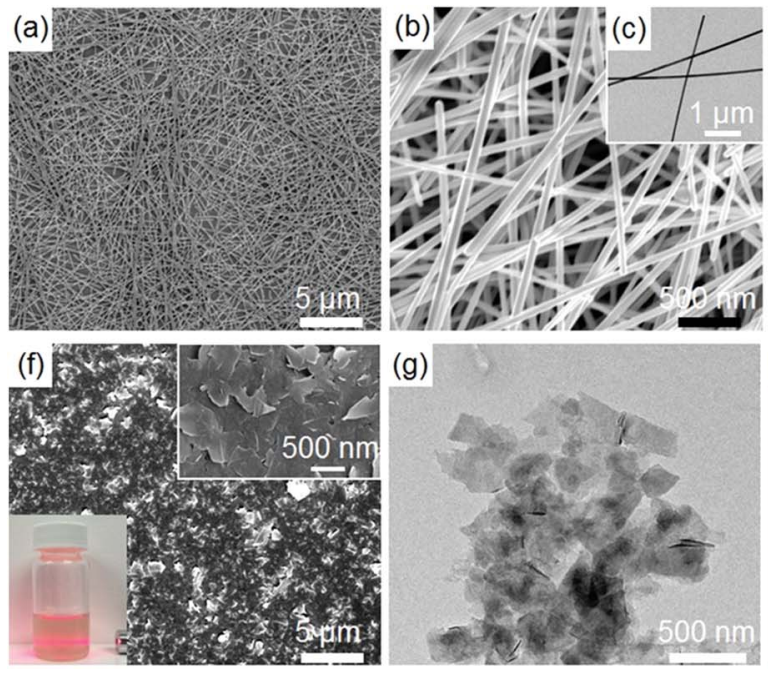

(g)

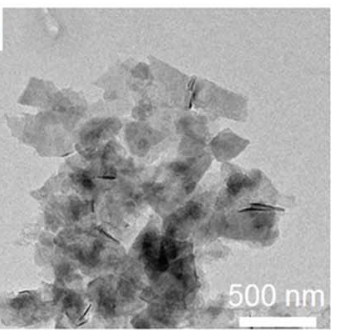

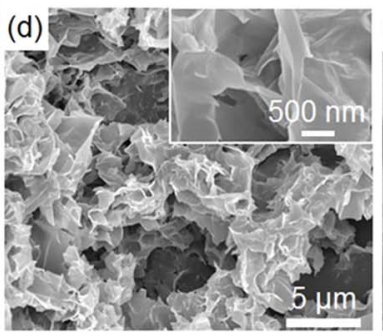
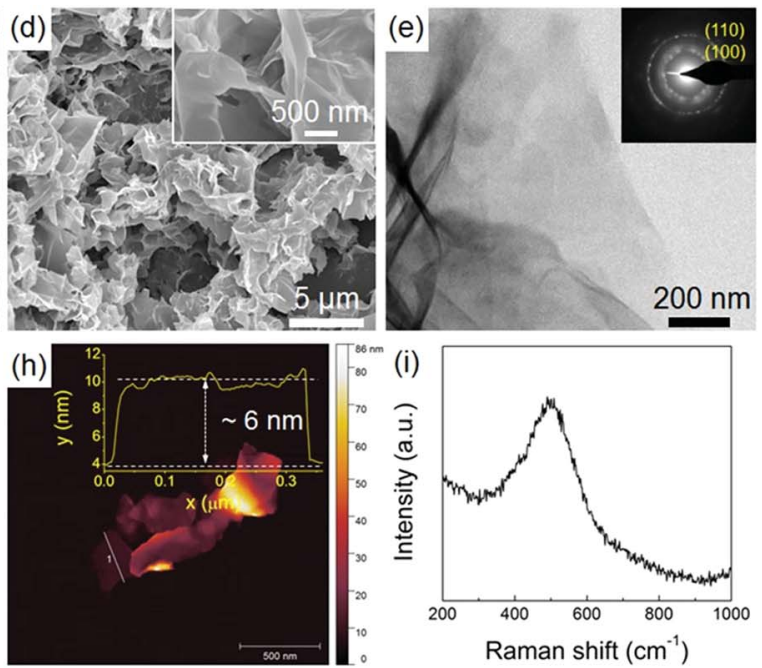

Fig. 1 SEM and TEM images of Ag NWs (a-c). SEM and TEM images of Ni(OH) $)_{2}$ SSs before $(d$ and e) and after ( $f$ and $g$ ) exfoliation. The inset in the lower-left corner of (f) is the photo of the exfoliated $\mathrm{Ni}(\mathrm{OH})_{2} \mathrm{NS}$ suspension. AFM image (h) with the corresponding height profile, and the Raman spectrum (i) of exfoliated $\mathrm{Ni}(\mathrm{OH})_{2} \mathrm{NSs}$. 

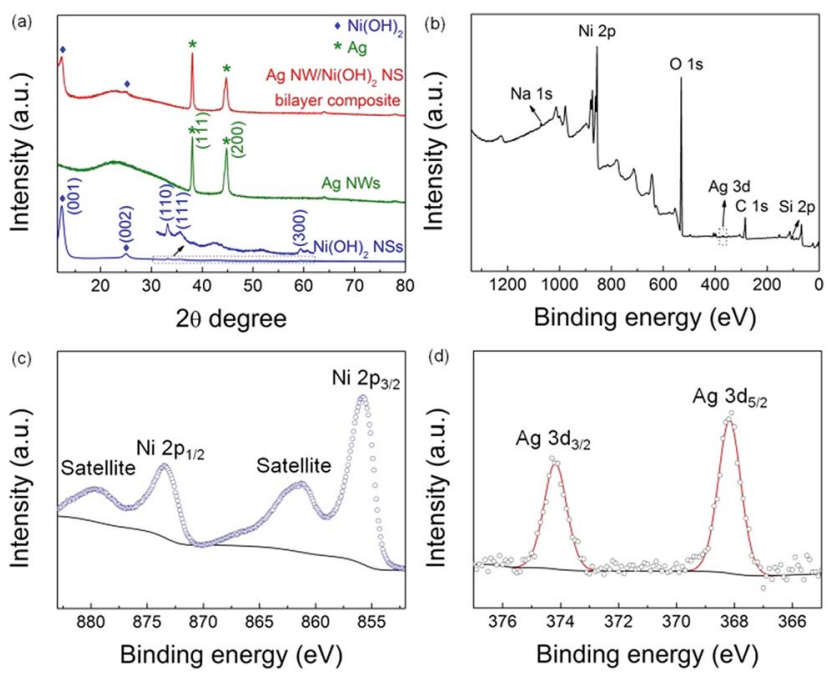

Fig. 2 XRD patterns (a) of $\mathrm{Ni}(\mathrm{OH})_{2} \mathrm{NSs}, \mathrm{Ag} \mathrm{NWs}$, and the Ag NW/ $\mathrm{Ni}(\mathrm{OH})_{2} \mathrm{NS}$ composite. XPS of the Ag NW/Ni(OH)$)_{2} \mathrm{NS}$ composite: survey scan (b), Ni 2p (c) and Ag 3d (d) spectra.

as silicon and sodium are from the glass substrate. The binding energies of $\mathrm{Ag} 3 \mathrm{~d}_{5 / 2}$ and $\mathrm{Ag} 3 \mathrm{~d}_{3 / 2}$ located at $\sim 368.2 \mathrm{eV}$ and $\sim 374.2 \mathrm{eV}$, respectively, correspond to metallic silver, ${ }^{17}$ and the $\mathrm{Ni} 2 \mathrm{p}_{3 / 2}$ located at $\sim 855.8 \mathrm{eV}$ is characteristic of $\mathrm{Ni}^{2+}$ in $\mathrm{Ni}(\mathrm{OH})_{2} \cdot{ }^{18}$

To study the effect of the $\mathrm{Ni}(\mathrm{OH})_{2}$ NS layer on the optical and electrical performances, Ag NW TEs with initial resistances of $\sim 245 \Omega \square^{-1}$ and $\sim 75 \Omega \square^{-1}$ were prepared first and then the $\mathrm{Ni}(\mathrm{OH})_{2}$ NSs were drop coated. The photographs (Fig. 3a) show that the $\mathrm{Ag} \mathrm{NW} / \mathrm{Ni}(\mathrm{OH})_{2} \mathrm{NS}$ electrodes have a high transparency. After coating with a small amount of the $\mathrm{Ni}(\mathrm{OH})_{2}$ NSs, the optical property (Fig. $3 \mathrm{~b}$ and c) is not compromised significantly because of the ultrathin NS structure.

Meanwhile, the $R_{\mathrm{s}}$ reduces by around $50 \%$ first, and then changes slightly with the increasing coating times (Fig. 3d). Fig. $4 \mathrm{a}-\mathrm{c}$ show the microstructures of the TEs before and after $\mathrm{Ni}(\mathrm{OH})_{2} \mathrm{NS}$ coating. The contacts between the $\mathrm{Ag} \mathrm{NWs}$ alone are very loose (Fig. 4a) thus the relatively large $R_{\mathrm{s}}$ cannot be avoided due to the weak electrical contacts and the empty voids in the NW network. After deposition of the $\mathrm{Ni}(\mathrm{OH})_{2}$ NS layer, the junction contacts and $R_{\mathrm{S}}$ value are greatly improved. The reduced $R_{\mathrm{S}}$ of the $\mathrm{Ag} \mathrm{NW} / \mathrm{Ni}(\mathrm{OH})_{2}$ NS composites should be attributed to the improvement of the NW junction contacts and the enhanced adhesion between the $\mathrm{Ag} \mathrm{NWs}, \mathrm{Ni}(\mathrm{OH})_{2} \mathrm{NSs}$ and the substrate. On the one hand, the $\mathrm{Ni}(\mathrm{OH})_{2} \mathrm{NSs}$ with different lateral areas can not only cover the NW junctions and infiltrate the empty spaces between the Ag NWs but also encapsulate the NW network as a coating layer (as shown in Fig. 4b-d), further increasing the conductive pathways by bridging a few unconnected nanowires. On the other hand, the NW contacts can be improved owing to the mechanical nanopressing effect ${ }^{5}$ of the $\mathrm{Ni}(\mathrm{OH})_{2}$ NSs and the capillary-force induced cold-welding. When the Ag NWs are covered by $\mathrm{Ni}(\mathrm{OH})_{2} \mathrm{NSs}$, the interfacial pressure can be a driving force to induce the welding effect at the NW junctions. In Fig. 4c, NW junctions with slightly compressed connections are clearly observed after coating with
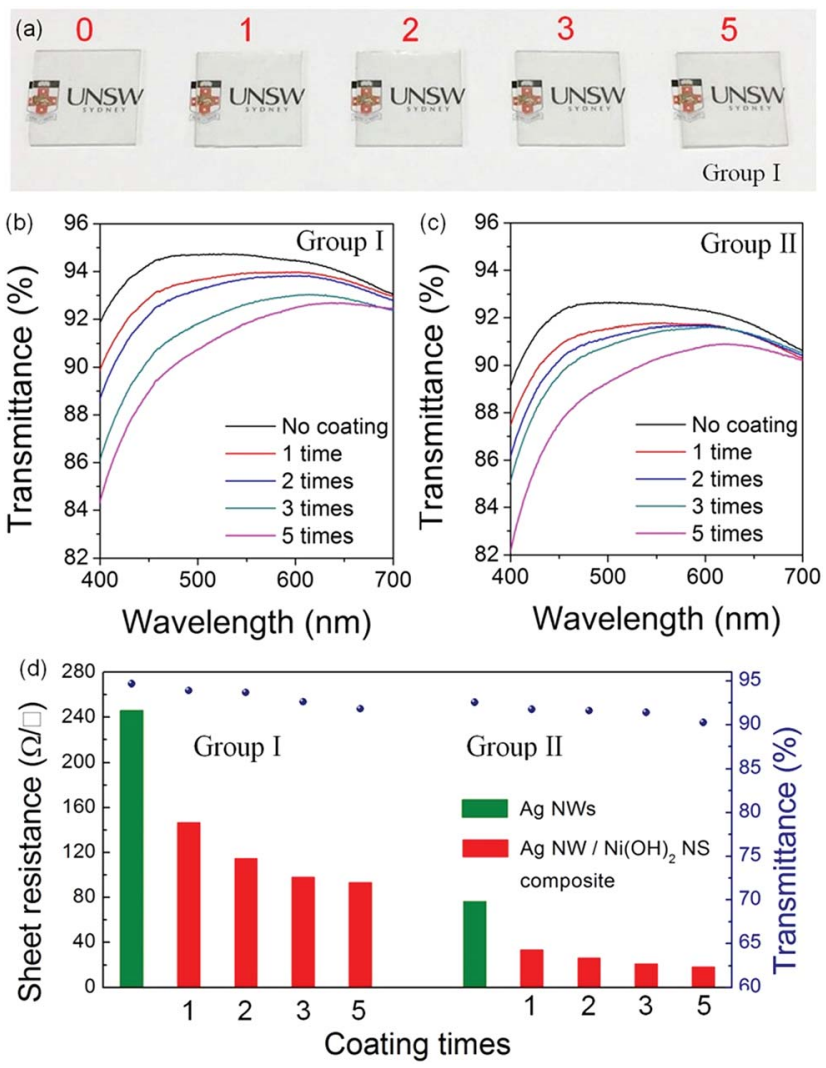

Fig. 3 Two groups of $\mathrm{Ag} \mathrm{NW} / \mathrm{Ni}(\mathrm{OH})_{2}$ TEs with different $\mathrm{Ni}(\mathrm{OH})_{2}$ coating times. The initial resistances of groups I and II are $\sim 245 \Omega \square^{-1}$ and $\sim 75 \Omega \square^{-1}$ respectively. Photographs (a) of group I. Optical transmittance (b and c) from UV-vis spectra of groups I and II. Sheet resistance and transmittance at $550 \mathrm{~nm}$ of the $\mathrm{Ag} \mathrm{NW} / \mathrm{Ni}(\mathrm{OH})_{2} \mathrm{NS}$ composite prepared on glass substrates with different coating times (d).

a $\mathrm{Ni}(\mathrm{OH})_{2} \mathrm{NS}$ layer. It has also been reported that capillary-force by solvent evaporation often induces cold-welding at a junction. Here, the solvent of the $\mathrm{Ni}(\mathrm{OH})_{2}$ NS solution is water and only

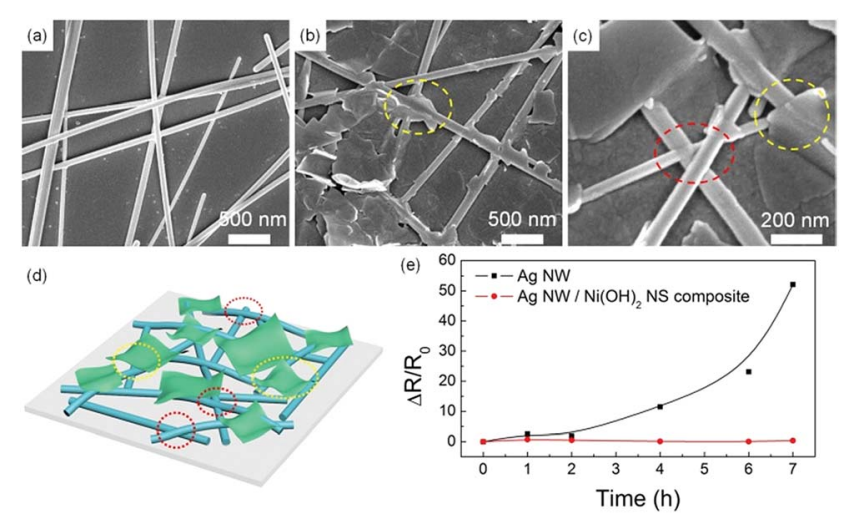

Fig. 4 SEM images of Ag NW (a) and Ag NW/Ni(OH) $)_{2} \mathrm{NS}$ (b and c) TEs. Schematic (d) of the $\mathrm{Ni}(\mathrm{OH})_{2}$ NSs covering the NW junctions (yellow dashed circles) and the compressed connections of NW junctions (red dashed circles). (e) Variation of $R_{\mathrm{s}}$ of $\mathrm{Ag} \mathrm{NW}$ and $\mathrm{Ag} \mathrm{NW} / \mathrm{Ni}(\mathrm{OH})_{2} \mathrm{NS}$ electrodes annealed at $200^{\circ} \mathrm{C}$ as a function of annealing time. $\Delta R=R$ $-R_{0}$, where $R$ and $R_{0}$ are the $R_{\mathrm{s}}$ value after annealing, and the original $R_{\mathrm{s}}$ value, respectively. 
a small amount of solution $(20 \mu \mathrm{L})$ is used for each coating. During the water evaporation process, the capillary-force, as a pull force, can bridge the separated NWs. ${ }^{19}$ Thus both mechanical nanopressing from the $\mathrm{Ni}(\mathrm{OH})_{2} \mathrm{NSs}$ and the coldwelding during water evaporation should be responsible for the strengthened NW contacts and enhanced conductivity. Moreover, with the increasing coating times the $R_{\mathrm{S}}$ decreases only slightly, indicating that the nanopressing and bridging effects become less effective as the hybrid network may have already reached an optimum state. To further evaluate the electrical and optical properties of the $\mathrm{Ag} \mathrm{NW} / \mathrm{Ni}(\mathrm{OH})_{2} \mathrm{NS}$ composite TEs, the $R_{\mathrm{S}}$ and transmittance at a wavelength of $550 \mathrm{~nm}$ were used to calculate the figure of merit (FoM) using the following equation defined by Haacke: ${ }^{20}$

$$
\Phi_{\mathrm{TC}}=T^{10} / R_{\mathrm{s}}
$$

The $\Phi_{\mathrm{TC}}$ value is FoM $19.3\left(10^{-3} \Omega^{-1}\right)$, based on the $R_{\mathrm{S}}$ of $18.56 \Omega \square^{-1}$ and the transmittance of $90.26 \%$, and this value is comparable to that of the commercial ITO (21.9).

Another issue with Ag NWs is their thermal stability. It is found that $\mathrm{Ag}$ NWs often undergo a spheroidization process during heating, transforming from nanowires to fragments or even nanoparticles due to the reduction of surface energy. Since the beginning of coalescence takes place at $200{ }^{\circ} \mathrm{C},{ }^{21} \mathrm{Ag}$ NWs and the $\mathrm{Ag} \mathrm{NW} / \mathrm{Ni}(\mathrm{OH})_{2}$ NS composite on the glass substrate were annealed at $200{ }^{\circ} \mathrm{C}$ for $7 \mathrm{~h}$ to evaluate their thermal stabilities. As shown in Fig. 4e, the $R_{\mathrm{s}}$ of the $\mathrm{Ag} \mathrm{NW}$ electrode increases around 50 fold, while the $\mathrm{Ag} \mathrm{NW} / \mathrm{Ni}(\mathrm{OH})_{2} \mathrm{NS}$ composite retains its original conductivity, and its resistance variation is very slight. According to the previous study, ${ }^{22}$ when annealing at $200{ }^{\circ} \mathrm{C}$ there is no significant decomposition or phase transition and the only change of $\alpha-\mathrm{Ni}(\mathrm{OH})_{2}$ is the removal of adsorbed and bonded water. Thus, the $\mathrm{Ni}(\mathrm{OH})_{2} \mathrm{NSs}$ act as a protective layer on the $\mathrm{Ag}$ NW electrode, effectively enhancing its thermal stability.

The as-synthesized $\mathrm{Ni}(\mathrm{OH})_{2}$ NSs were dispersed on carbon

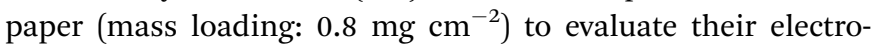
chemical performance. As shown in Fig. 5a, the cyclic voltammogram (CV) curves show a pair of redox peaks corresponding to the transition between $\mathrm{Ni}(\mathrm{OH})_{2}$ and $\mathrm{NiOOH}$, indicating that the current response is governed by the faradaic redox reaction. It is known that the electrochemical behaviour can be evaluated by $i=a \nu^{b}{ }^{23}$ in which $b \approx 0.5$ corresponds to the semi-infinite linear diffusion controlled current of a battery material, whereas $b \approx 1$ corresponds to the surface controlled current of a pseudocapacitor material. ${ }^{24}$ Here, the linear relationship (Fig. 5b) between the peak current and the square root of the scan rate demonstrates that $\mathrm{Ni}(\mathrm{OH})_{2}$ is a battery-type material. Afterwards, the symmetric all-solid-state supercapacitors were fabricated by assembling the $\mathrm{Ag} \mathrm{NW} / \mathrm{Ni}(\mathrm{OH})_{2}$ NS composite with PVA/KOH as the solid-state electrolyte, and the CV scan was conducted over a potential window from 0 to $0.8 \mathrm{~V}$. As shown in Fig. $5 c$, the flattened curve for the Ag NW film indicates that the capacitance of $\mathrm{Ag} \mathrm{NW}$ is negligible while the $\mathrm{Ag} \mathrm{NW} / \mathrm{Ni}(\mathrm{OH})_{2} \mathrm{NS}$ composite shows a much higher current density, which demonstrates the capacitive contribution of the $\mathrm{Ni}(\mathrm{OH})_{2} \mathrm{NSs}$.

Fig. 5d shows the $\mathrm{CV}$ plots of the $\mathrm{Ag} \mathrm{NW} / \mathrm{Ni}(\mathrm{OH})_{2}$ NS composite at scan rates gradually increasing from 5 to
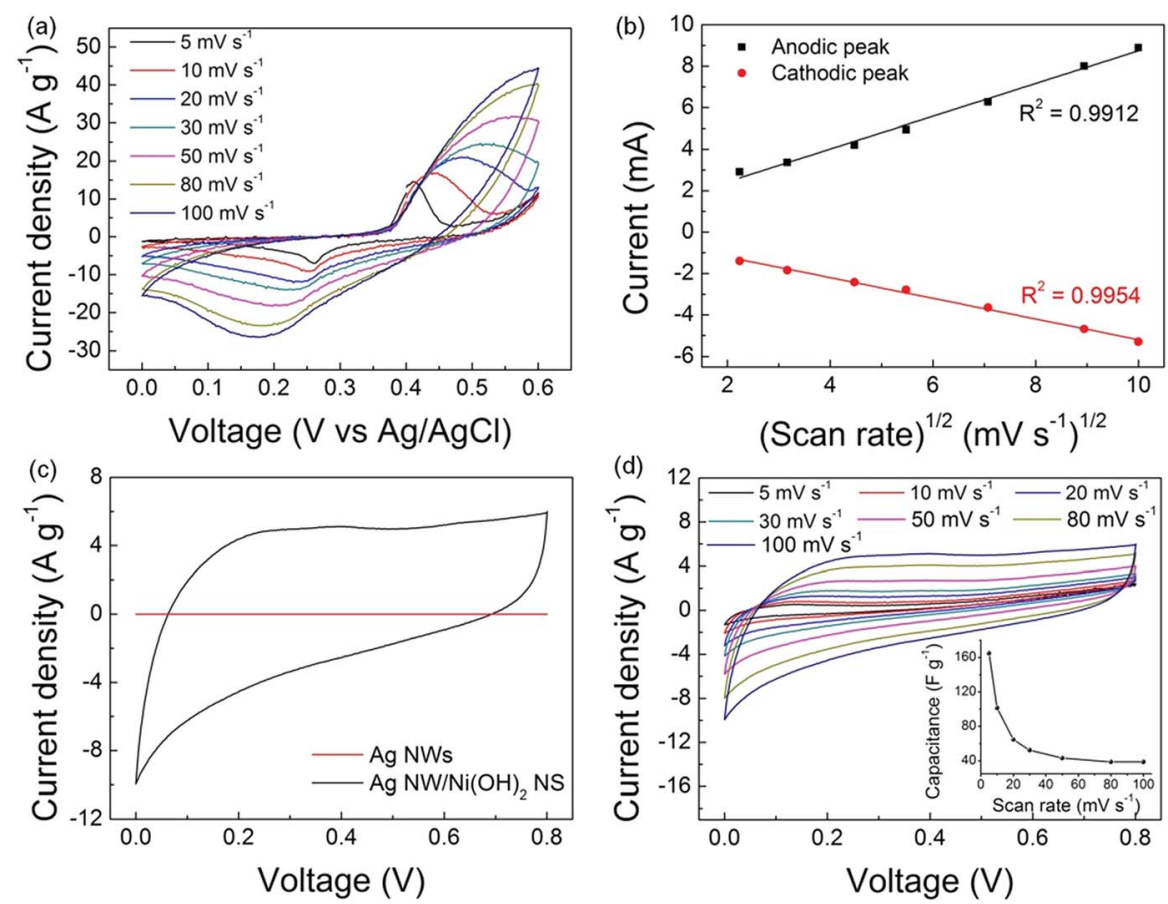

Fig. $5 \mathrm{CV}$ curves (a) of $\mathrm{Ni}(\mathrm{OH})_{2} \mathrm{NSs}$ in $1 \mathrm{M} \mathrm{KOH}$ electrolyte at different scan rates, and (b) the relationship between the peak currents and the square root of the corresponding scan rates in the CV curves. CV curves (c) of Ag NW and Ag NW/Ni(OH) $2 \mathrm{NS}$ symmetric all-solid-state supercapacitors using glass substrates; the scan rate is $100 \mathrm{mV} \mathrm{s}^{-1}$. CV curves (d) of Ag NW/Ni(OH) 2 NS composite all-solid-state supercapacitors on glass substrates at different scan rates; inset: the specific capacitance. 
$100 \mathrm{mV} \mathrm{s}^{-1}$. The quasi-rectangular shape of the $\mathrm{CV}$ curve is preserved and becomes more obvious with the increasing scan rates, suggesting a fast charge diffusion and stable capacitive performance. Meanwhile, the increased area is a result of the efficient ion diffusion in the polymer electrolyte. ${ }^{25}$ The specific capacitance can be calculated from the CV curves by using the equation:

$$
C_{\mathrm{sp}}=\frac{\int I \mathrm{~d} V}{2 \nu m \Delta V}
$$

where $C_{\mathrm{sp}}$ is the specific capacitance $\left(\mathrm{F} \mathrm{g}^{-1}\right), I$ is the current, $\nu$ is the scan rate $\left(\mathrm{V} \mathrm{s}^{-1}\right), m$ is the mass of supercapacitive material and $\Delta V$ is the potential window. It can be seen that the capacitance decreases from $164.8 \mathrm{~F} \mathrm{~g}^{-1}$ to $38.9 \mathrm{~F} \mathrm{~g}^{-1}$ when the scan rate is gradually increased (inset of Fig. 5d).

Given that both the $\mathrm{Ag}$ NW network and $\mathrm{Ni}(\mathrm{OH})_{2}$ NSs have superior mechanical flexibility, a flexible supercapacitor was fabricated using a similar procedure except that it was transferred onto a PDMS substrate. The $\mathrm{Ag} \mathrm{NW} / \mathrm{Ni}(\mathrm{OH})_{2}$ NS flexible supercapacitor is semi-transparent because of the transparent nature of the PDMS substrate, as shown by the photograph and the UV-vis spectrum (Fig. 6a). Fig. 6b shows a series of CV measurements of the flexible supercapacitor under different potential windows ranging from 0 to $1 \mathrm{~V}$. It can be seen that the supercapacitive performance is stable, while the large peak current corresponding to the faradaic reaction becomes more obvious with the increasing working potential to $1 \mathrm{~V}$. Similar to the supercapacitor using the glass substrate, the CVs of the flexible device recorded at different scan rates (Fig. 6c) become a more symmetrical rectangular shape without obvious distortion, implying a good reversibility and a fast charge-discharge property. With the increasing scan rates, the current (Fig. 6d) shows a linear response $(i-\nu)$, typical of a capacitor. ${ }^{24}$ Moreover, the supercapacitor performance is characterized by the galvanostatic charge-discharge (GCD) curves at different current densities (Fig. 6e). Unlike EDLCs which possess a linear voltage-time relationship, the asymmetric GCD curve of the Ag $\mathrm{NW} / \mathrm{Ni}(\mathrm{OH})_{2}$ NS supercapacitor is indicative of pseudocapacitive behaviour due to the redox reaction. ${ }^{26}$ Accordingly, the specific capacitance can be calculated by the following equation:

$$
C_{\mathrm{sp}}=\frac{I \times t}{m \Delta V}
$$

where $C_{\mathrm{sp}}$ is the specific capacitance $\left(\mathrm{F} \mathrm{g}^{-1}\right), I$ is the chargedischarge current, $t$ is the discharge time, $m$ is the mass of active material and $\Delta V$ is the potential change during the discharge process. The specific capacitance (Fig. 6f) is $\sim 78 \mathrm{~F} \mathrm{~g}^{-1}$ at $0.5 \mathrm{~A} \mathrm{~g}^{-1}$, which is comparable to the value of previously reported all-solid-state flexible supercapacitors. ${ }^{27,28}$ The cycling stability of the $\mathrm{Ag} \mathrm{NW} / \mathrm{Ni}(\mathrm{OH})_{2}$ NS flexible supercapacitor was also investigated, as shown in Fig. 6g. For the initial 200 cycles, the specific capacitance increases slightly due to the increased contact area between the electrode and solid-state electrolyte, corresponding to an activation process of the electrode. Afterwards, the capacitance starts to decline and retains a level of $62.5 \%$ after 1000 cycles which decreases to $45.5 \%$ after 1500 cycles. This retention loss is attributed to the capacitance degradation of $\mathrm{Ni}(\mathrm{OH})_{2}$ caused by phase transformation during the charge-discharge processes. ${ }^{29}$ The performance degradation is also characterized by the electrochemical impedance spectroscopy result (Fig. S1†), in which the charge transfer resistance $\left(R_{\mathrm{ct}}\right)$ increases after the cycling test due to the degradation of $\mathrm{Ni}(\mathrm{OH})_{2}$. Furthermore, the CV curves of the flexible supercapacitor under flat, bent and even twisted conditions were obtained (Fig. 6h). It can be seen that the shape of the CV curves under bent and twisted conditions is similar to that obtained for the original state in spite of the slight degradation. The discharge property and capacitance retention with
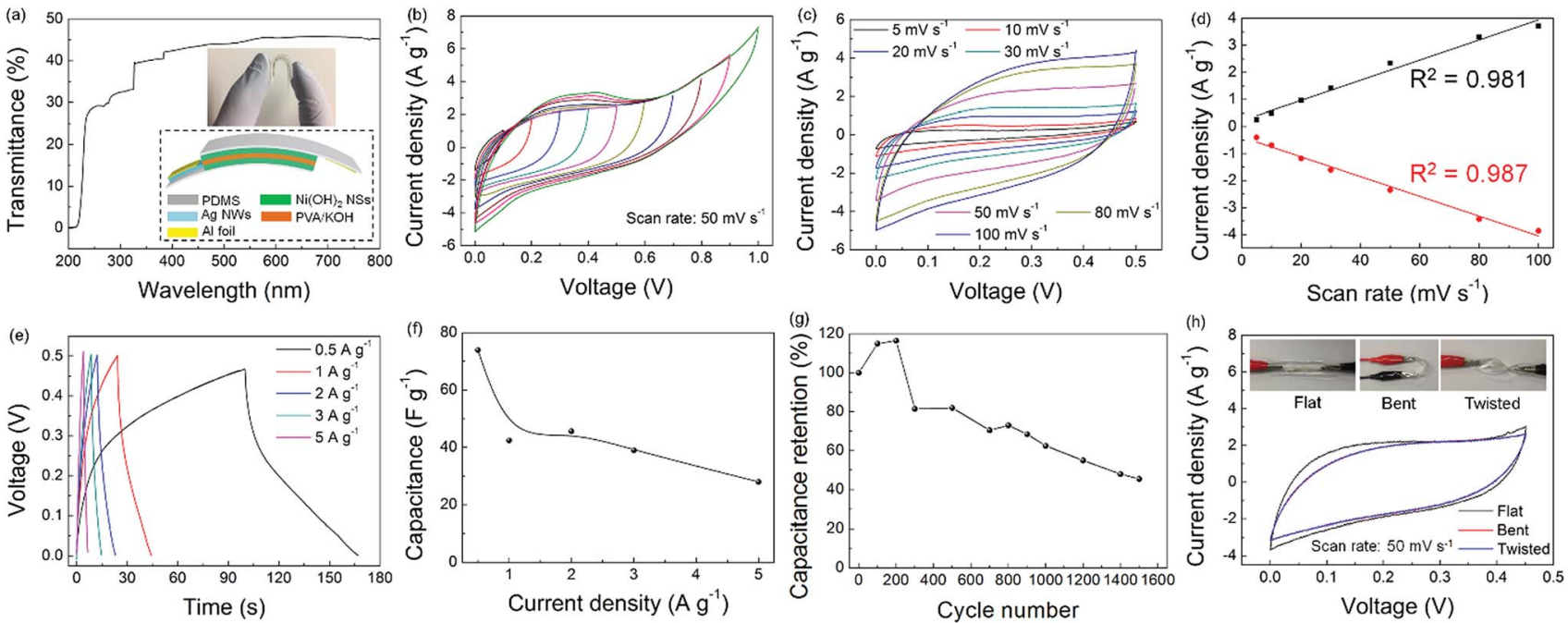

Fig. 6 Schematic, photograph and UV-vis spectrum (a) of the Ag NW/Ni(OH) $)_{2}$ NS flexible supercapacitor. CV curves at different potential windows (b) and different scan rates (c). The relationship between current density and the corresponding scan rates in CV curves (d). GCD curves (e) and the corresponding capacitance (f) at different current densities. Capacitance retention (g) by a cycling test at $5 \mathrm{~A} \mathrm{~g}^{-1}$. CV curves (h) under flat, bent and twisted conditions. The insets are the photographs of the corresponding conditions. 
increasing bending times (bending angle: $180^{\circ}$ ) are also shown in Fig. S2. $\dagger$ After 300 times of bending, the capacitance drops to $\sim 54 \%$ of its initial value, indicating a potential for flexible applications.

\section{Conclusions}

In summary, we have demonstrated a composite based on $\mathrm{Ag}$ nanowires (NWs) and $\mathrm{Ni}(\mathrm{OH})_{2}$ nanosheets (NSs) for transparent electrode and all-solid-state supercapacitor applications. The $\mathrm{Ni}(\mathrm{OH})_{2}$ NSs were used not only to infiltrate the empty spaces between the Ag NWs and improve the junction resistance but also to protect the $\mathrm{Ag}$ NW network against thermal degradation. As a result, a composite with a sheet resistance of $18.56 \Omega \square^{-1}$, a transmittance of $90.26 \%$ as well as an improved thermal stability was successfully prepared after simply coating the $\mathrm{Ag}$ NWs with $\mathrm{Ni}(\mathrm{OH})_{2}$ NSs. Moreover, taking the high conductivity of $\mathrm{Ag}$ NWs and the pseudocapacitive behaviour of $\mathrm{Ni}(\mathrm{OH})_{2} \mathrm{NS}$ into consideration, the $\mathrm{Ag} \mathrm{NW} / \mathrm{Ni}(\mathrm{OH})_{2} \mathrm{NS}$ composite was designed to fabricate an all-solid-state flexible supercapacitor. The supercapacitor device exhibited a good capacitance, moderate cycle stability and flexibility, indicating that the composite could be regarded as a promising candidate for flexible supercapacitors.

\section{Conflicts of interest}

There are no conflicts to declare.

\section{Acknowledgements}

This work is funded by the Australian Research Council Project (grant no. FT140100032). The authors would like to thank Dr Yin Yao for assistance with AFM measurements.

\section{References}

1 S. Ye, A. R. Rathmell, Z. Chen, I. E. Stewart and B. J. Wiley, Adv. Mater., 2014, 26, 6670-6687.

2 D. Y. Choi, H. W. Kang, H. J. Sung and S. S. Kim, Nanoscale, 2013, 5, 977-983.

3 R. Chen, S. R. Das, C. Jeong, M. R. Khan, D. B. Janes and M. A. Alam, Adv. Funct. Mater., 2013, 23, 5150-5158.

4 K. Zilberberg, F. Gasse, R. Pagui, A. Polywka, A. Behrendt, S. Trost, R. Heiderhoff, P. Görrn and T. Riedl, Adv. Funct. Mater., 2014, 24, 1671-1678.

5 H. Sohn, S. Kim, W. Shin, J. M. Lee, H. Lee, D.-J. Yun, K.-S. Moon, I. T. Han, C. Kwak and S.-J. Hwang, ACS Appl. Mater. Interfaces, 2018, 10, 2688-2700.
6 X. Y. Zeng, Q. K. Zhang, R. M. Yu and C. Z. Lu, Adv. Mater., 2010, 22, 4484-4488.

7 Y. Jin, K. Wang, Y. Cheng, Q. Pei, Y. Xu and F. Xiao, ACS Appl. Mater. Interfaces, 2017, 9, 4733-4741.

8 Q. Zhao, M. Zhao, J. Qiu, W. Y. Lai, H. Pang and W. Huang, Small, 2017, 13, 1701091.

9 J.-L. Xu, Y.-H. Liu, X. Gao, Y. Sun, S. Shen, X. Cai, L. Chen and S.-D. Wang, ACS Appl. Mater. Interfaces, 2017, 9, 2764927656.

10 V. Augustyn, P. Simon and B. Dunn, Energy Environ. Sci., 2014, 7, 1597-1614.

11 H. Wang, H. S. Casalongue, Y. Liang and H. Dai, J. Am. Chem. Soc., 2010, 132, 7472-7477.

12 X. Zhao, X. Ding, Y. Xia, X. Jiao and D. Chen, ACS Appl. Nano Mater., 2018, 1, 1476-1483.

13 X. Xiong, D. Ding, D. Chen, G. Waller, Y. Bu, Z. Wang and M. Liu, Nano Energy, 2015, 11, 154-161.

14 H. Du, T. Wan, B. Qu, F. Cao, Q. Lin, N. Chen, X. Lin and D. Chu, ACS Appl. Mater. Interfaces, 2017, 9, 20762-20770.

15 M. W. Louie and A. T. Bell, J. Am. Chem. Soc., 2013, 135, 12329-12337.

16 Y. Zhu, C. Cao, S. Tao, W. Chu, Z. Wu and Y. Li, Sci. Rep., 2014, 4, 5787.

17 E. Stathatos, P. Lianos, P. Falaras and A. Siokou, Langmuir, 2000, 16, 2398-2400.

18 N. McIntyre and M. Cook, Anal. Chem., 1975, 47, 2208-2213.

19 K. Zhang, J. Li, Y. Fang, B. Luo, Y. Zhang, Y. Li, J. Zhou and B. Hu, Nanoscale, 2018, 10, 12981-12990.

20 G. Haacke, J. Appl. Phys., 1976, 47, 4086-4089.

21 D. Chen, J. Liang, C. Liu, G. Saldanha, F. Zhao, K. Tong, J. Liu and Q. Pei, Adv. Funct. Mater., 2015, 25, 7512-7520.

22 L. Xu, Y.-S. Ding, C.-H. Chen, L. Zhao, C. Rimkus, R. Joesten and S. L. Suib, Chem. Mater., 2008, 20, 308-316.

23 H. Lindström, S. Södergren, A. Solbrand, H. Rensmo, J. Hjelm, A. Hagfeldt and S.-E. Lindquist, J. Phys. Chem. B, 1997, 101, 7717-7722.

24 V. Augustyn, J. Come, M. A. Lowe, J. W. Kim, P.-L. Taberna, S. H. Tolbert, H. D. Abruña, P. Simon and B. Dunn, Nat. Mater., 2013, 12, 518.

25 W. Xiong, X. Hu, X. Wu, Y. Zeng, B. Wang, G. He and Z. Zhu, J. Mater. Chem. A, 2015, 3, 17209-17216.

26 J. Liu, L. Zhang, H. B. Wu, J. Lin, Z. Shen and X. W. D. Lou, Energy Environ. Sci., 2014, 7, 3709-3719.

27 W. Liu, X. Li, M. Zhu and X. He, J. Power Sources, 2015, 282, 179-186.

28 T. Gu and B. Wei, J. Mater. Chem. A, 2016, 4, 12289-12295.

29 J. Zhang, S. Liu, G. Pan, G. Li and X. Gao, J. Mater. Chem. A, 2014, 2, 1524-1529. 